\title{
Periodicity, almost periodicity for time scales and related functions
}

DOI 10.1515/msds-2016-0003

Received December 9, 2015; accepted May 4, 2016

Abstract: In this paper, we study almost periodic and changing-periodic time scales considered by Wang and Agarwal in 2015. Some improvements of almost periodic time scales are made. Furthermore, we introduce a new concept of periodic time scales in which the invariance for a time scale is dependent on an translation direction. Also some new results on periodic and changing-periodic time scales are presented.

Keywords: Time scales, Almost periodic time scales, Changing periodic time scales

MSC: 26E70, 43A60, 34N05.

\section{Introduction}

Recently, many papers were devoted to almost periodic issues on time scales (see for examples [1-5]). In 2014, to study the approximation property of time scales, Wang and Agarwal introduced some new concepts of almost periodic time scales in [6] and the results show that this type of time scales can not only unify the continuous and discrete situations but can also strictly include the concept of periodic time scales. In 2015, some open problems related to this topic were proposed by the authors (see [4]).

Wang and Agarwal addressed the concept of changing-periodic time scales in 2015. This type of time scales can contribute to decomposing an arbitrary time scales with bounded graininess function $\mu$ into a countable union of periodic time scales i.e, Decomposition Theorem of Time Scales.

The concept of periodic time scales which appeared in $[7,8]$ can be quite limited for the simple reason that it requires inf $\mathbb{T}=-\infty$ and $\sup \mathbb{T}=+\infty$. The first concepts of periodic time scales and periodic functions were proposed by Kaufmann and Raffoul in [7], and they were improved by Adivar in [9]. However, the concepts of periodic time scales proposed by [7-9] were without considering the shift direction of time scales, which will be quite limited for understanding the recent results from $[2,10]$ etc. For an example, consider

$$
\mathbb{T}=\bigcup_{k=0}^{+\infty}[2 k, 2 k+1]
$$

according to the concept of periodic time scales from [7, 8], (1.1) is not a periodic time scale since inf $\mathbb{T}=0$. In fact, for any $t \in \mathbb{T}$, we have $t+2 \in \mathbb{T}$, but there exists $t_{0}=0 \in \mathbb{T}$ such that $t_{0}-2=-2 / \in \mathbb{T}$. Nevertheless, (1.1) has very nice closedness in translation for time variables if the time scale is only translated towards the positive direction, because for any $t \in \mathbb{T}$, we have $t+2 \in \mathbb{T}$. For another example, consider

$$
\mathbb{T}=\overline{\bigcup_{n \in \mathbb{Z}^{+} \cup\{0\}}\left[2^{2 n}, 2^{2 n+1}\right]}
$$

Chao Wang: Department of Mathematics, Yunnan University, Kunming, Yunnan 650091, People’s Republic of China, E-mail: chaowang@ynu.edu.cn

Ravi P. Agarwal: Department of Mathematics, Texas A\&M University-Kingsville, TX 78363-8202, Kingsville, TX, USA, E-mail: Agarwal@tamuk.edu

Donal 0'Regan: School of Mathematics, Statistics and Applied Mathematics, National University of Ireland, Galway, Ireland, E-mail: donal.oregan@nuigalway.ie 
according to the new concept of periodic time scales from [9], (1.2) is also not a periodic time scales since $\mathbb{T}$ has a infimum that equals to 1 with respect to the shift operator $\delta_{-}$. In fact, for any $t \in \mathbb{T}$, although $\delta_{+}(4, t)=4 t \in$ $\mathbb{T}$, there exists $t_{0}=1 \in \mathbb{T}$ such that $\delta_{-}\left(4, t_{0}\right)=t_{0} / 4=1 / 4 / \in \mathbb{T}$. However, (1.2) also has very nice closedness in shift for time variables if the time scale is only shifted towards the positive direction, because for any $t \in \mathbb{T}$, we have $\delta_{+}(4, t) \in \mathbb{T}$. Hence, concepts of periodic time scales from [7, 9] were without considering the shift direction of time scales.

In this paper we propose a new concept of periodic time scales with "translation direction" so that we can regard (1.1) as a periodic time scale. Also we improve the results on almost periodic time scales from [6]. In addition, we obtain new results related to changing-periodic time scales from [2].

\section{Periodic and almost periodic time scales}

To obtain some new results related to periodic and almost periodic time scales, first, we introduce some new definitions of intersection type for a translation time scale.

Let

$$
\Pi_{1}:=\left\{\tau \in \mathbb{R}: \mathbb{T} \cap \mathbb{T}^{\tau} \neq \emptyset\right\} \neq\{0\},
$$

where $\mathbb{T}^{\tau}:=\mathbb{T}+\tau=\{t+\tau: \forall t \in \mathbb{T}\}$.

Definition 2.1. We introduce three types of intersection for a translation time scale as follows:

(1) We say $\mathbb{T}$ is a positive-direction intersection time scale if for any $p>0$, there exists a number $P>p$ and $P \in \Pi_{1}$, we call $\mathbb{T} \cap \mathbb{T}^{P}$ the positive-direction intersection for $\mathbb{T}$.

(2) We say $\mathbb{T}$ is a negative-direction intersection time scale if for any $q<0$, there exists a number $Q<q$ and $Q \in \Pi_{1}$, we call $\mathbb{T} \cap \mathbb{T}^{Q}$ the negative-direction intersection for $\mathbb{T}$.

(3) We say $\mathbb{T}$ is a bi-direction intersection time scale if for any $p>0$ and $q<0$, there exists two numbers $P>p, Q<q$ and $P, Q \in \Pi_{1}$, we call $\left(\mathbb{T} \cap \mathbb{T}^{P}\right) \cup\left(\mathbb{T} \cap \mathbb{T}^{Q}\right)$ the bi-direction intersection for $\mathbb{T}$.

(4) We say $\mathbb{T}$ is an oriented-direction intersection time scale if $\mathbb{T}$ is a positive-direction intersection time scale or a negative-direction intersection time scale.

Remark 2.1. From Definition 2.1, we obtain that if $\sup \mathbb{T}=+\infty$, inf $\mathbb{T}=-\infty$, then $\mathbb{T}$ is a bi-direction intersection time scale since for any $\tau \in \mathbb{R}$, we have $\mathbb{T} \cap \mathbb{T}^{\tau}=\{-\infty,+\infty\}$. If $\inf \mathbb{T}=-\infty$, then $\mathbb{T}$ is a negative-direction intersection time scale since for any $\tau \in \mathbb{R}$, we have $\mathbb{T} \cap \mathbb{T}^{\tau}=\{-\infty\}$. If sup $\mathbb{T}=+\infty$, then $\mathbb{T}$ is a positive-direction intersection time scale since for any $\tau \in \mathbb{R}$, we have $\mathbb{T} \cap \mathbb{T}^{\tau}=\{+\infty\}$.

Next, we introduce a new concept of periodic time scales. First we recall the following definition from the literature.

Definition 2.2 ([8]). A time scale $\mathbb{T}$ is called a periodic time scale if

$$
\Pi:=\{\tau \in \mathbb{R}: t \pm \tau \in \mathbb{T}, \forall t \in \mathbb{T}\} \neq\{0\} .
$$

Note that $\mathbb{T}$ defined in Definition 2.2 is a particular bi-direction intersection time scale according to (3) in Definition 2.1. In fact, for any $\tau \in \Pi$, we have $\mathbb{T} \cap \mathbb{T}^{ \pm \tau}=\mathbb{T}$.

We now consider some new concepts which are more general than Definition 2.2.

Definition 2.3. We say $\mathbb{T}$ is called a periodic time scale if

$$
\Pi_{2}:=\left\{\tau \in \mathbb{R}: \mathbb{T}^{\tau} \subseteq \mathbb{T}, \forall t \in \mathbb{T}\right\} \neq\{0\} .
$$

Furthermore, we can describe it in detail as follows: 
(a) if for any $p>0$, there exists a number $P>p$ and $P \in \Pi_{2}$, we say $\mathbb{T}$ is a positive-direction periodic time scale;

(b) if for any $q<0$, there exists a number $Q<q$ and $Q \in \Pi_{2}$, we say $\mathbb{T}$ is a negative-direction periodic time scale.

(c) if $\pm \tau \in \Pi_{2}$, we say $\mathbb{T}$ is a bi-direction periodic time scale;

(d) we say $\mathbb{T}$ is an oriented-direction periodic time scale if $\mathbb{T}$ is a positive-direction periodic time scale or a negative-direction periodic time scale.

Remark 2.2. From Definition 2.3, we obtain that a bi-direction periodic time scale is an oriented-direction periodic time scale. Furthermore, one can easily observe that Definition 2.2 is just a concept of bi-direction periodic time scales, it is just a particular case of Definition 2.3. Under Definition 2.3, (1.1) is a positive-direction periodic time scale.

From Definitions 2.1 and 2.3, and from Zorn's Lemma, we obtain a sufficient condition to guarantee that $\mathbb{T}$ is an oriented-direction periodic time scale.

Lemma 2.1 ([11], Zorn's Lemma). Suppose $(P, \preceq)$ is a partially ordered set. Suppose a non-empty partially ordered set $P$ has the property that every non-empty chain has an upper bound in $P$. Then the set $P$ contains at least one maximal element.

Theorem 2.1. Let $\mathbb{T}$ be an oriented-direction intersection time scale. If for any given $\varepsilon>0$, there exists a constant $l(\varepsilon)$ such that each interval of length $l(\varepsilon)$ contains a $\tau(\varepsilon) \in \Pi_{1}$ such that

$$
d\left(\mathbb{T}, \mathbb{T}_{\tau}\right)<\varepsilon,
$$

that is, for any $\varepsilon>0$, the following set

$$
E\{\mathbb{T}, \varepsilon\}:=\left\{\tau \in \Pi_{1}: d\left(\mathbb{T}, \mathbb{T}_{\tau}\right)<\varepsilon\right\}
$$

is relatively dense in $\Pi_{1}$, where $d(\cdot, \cdot)$ denote a Hausdorff distance and $\mathbb{T}_{\tau}=\mathbb{T} \cap \mathbb{T}^{\tau}$. Then $\mathbb{T}$ is an orienteddirection periodic time scale.

Proof. Note that from the condition of Theorem 2.1, we obtain $\mathbb{T}_{\tau} \subset \mathbb{T}$, and let $L:=\sup _{n \in \mathbb{N}}\left\{l\left(\frac{1}{n}\right)\right\}$. Without loss of generality, we assume that $\mathbb{T}$ is a positive-direction intersection time scale, and then for any $p>0$, there exists some $n_{0}>0$ such that $n_{0} L>p$. So we can choose a sequence $\left\{\tau_{n}\right\}_{n=1}^{\infty} \subset\left[p, n_{0} L\right]$ such that

$$
d\left(\mathbb{T}, \mathbb{T}_{\tau_{n}}\right)<\frac{1}{n} .
$$

We denote the set

$$
\mathbb{I}=\left\{\mathbb{T}_{\tau}: \tau \in\left[p, n_{0} L\right]\right\},
$$

and obviously, I is a closed set. From (2.4), we obtain the totally ordered set $\left\{\mathbb{T}_{\tau_{n}} \in \mathbb{I}: \mathbb{T}_{\tau_{n}} \subset \mathbb{T}_{\tau_{n+1}}, n \in \mathbb{N}\right\}$ and $\lim _{n \rightarrow \infty} \mathbb{T}_{\tau_{n}}=\mathbb{T}_{\tau_{\infty}}=\mathbb{T} \in \mathbb{I}$. Hence, $\mathbb{T}$ is the maximum element in $\mathbb{I}$.

Now $\mathbb{I}$ forms a semi-ordered set with respect to the inclusion relation and $\mathbb{I}$ is closed. Denote $\mathbb{I}^{\star}$ an arbitrary subset of $\mathbb{I}$ and is totally ordered. We consider two cases:

Case (1):

$$
\mathbb{I}^{\star}=\left\{\mathbb{T}_{\tau_{n}} \in \mathbb{I}: \mathbb{T}_{\tau_{n}} \supset \mathbb{T}_{\tau_{n+1}}, n \in \mathbb{N}\right\},
$$

then $\mathbb{T}_{\tau_{1}} \in \mathbb{I}^{\star} \subset \mathbb{I}$ and $\mathbb{T}_{\tau_{1}}$ is an upper bound of $\mathbb{I}^{*}$ in $\mathbb{I}$.

Case (2):

$$
\mathbb{I}^{\star}=\left\{\mathbb{T}_{\tau_{n}} \in \mathbb{I}: \mathbb{T}_{\tau_{n}} \subset \mathbb{T}_{\tau_{n+1}}, n \in \mathbb{N}\right\},
$$

then $\lim _{n \rightarrow \infty} \mathbb{T}_{\tau_{n}}=\bigcup_{n=1}^{\infty} \mathbb{T}_{\tau_{n}}=\mathbb{T}_{\tau_{\infty}}$ is an upper bound of $\mathbb{I}^{\star}$. Because $\mathbb{I}$ is closed, then $\mathbb{T}_{\tau_{\infty}} \in \mathbb{I}$. According to Zorn's Lemma (i.e., Lemma 2.1), there exists some $\tau_{0} \in\left[p, n_{0} L\right]$ such that $\mathbb{T} \cap \mathbb{T}^{\tau_{0}}:=\mathbb{T}_{\tau_{0}}$ is the maximum element in $\mathbb{I}$. 
From the above, we obtain that $\mathbb{T} \cap \mathbb{T}^{\tau_{0}}$ and $\mathbb{T}$ are two maximum elements in $\mathbb{I}$. Therefore, it follows from $\mathbb{T} \cap \mathbb{T}^{\tau_{0}} \subseteq \mathbb{T}$ that $\mathbb{T} \cap \mathbb{T}^{\tau_{0}}=\mathbb{T}$, i.e., $\mathbb{T}$ is an oriented-direction periodic time scale with $\tau_{0}$-period. This completes the proof.

Remark 2.3. In [12], to have $\mathbb{T} \cap \mathbb{T}^{\tau} \neq \emptyset$, the authors changed the time scale $\mathbb{T}^{\tau}$ in Definition 8 from [6] into $\mathbb{T}_{\tau}$, which will lead to an oriented-direction periodicity for a time scale according to Theorem 2.1.

Remark 2.4. From Theorem 2.1, we see that Lemmas 10, 11, Theorems 12, 13, 14 and Corollary 15 in [12] are based on oriented-direction periodic time scales.

Example 2.1. Let $a>1$ and consider the following almost periodic time scale

$$
\mathbb{P}_{a,|\sin \sqrt{3} t+\sin \sqrt{7} t|}=\bigcup_{m=1}^{\infty}\left[p_{m}, a+p_{m}\right]
$$

where

$$
\begin{aligned}
& p_{m}=(m-1) a+\sum_{k=1}^{m-1} \mid \sin \sqrt{3}(k a+|\sin \sqrt{3} a+\sin \sqrt{7} a| \\
& +\mid \sin \sqrt{3}(2 a+|\sin \sqrt{3} a+\sin \sqrt{7} a|) \\
& +\sin \sqrt{7}(2 a+|\sin \sqrt{3} a+\sin \sqrt{7} a|) \mid \\
& +\ldots+\mid \sin \sqrt{3}((k-1) a+|\sin \sqrt{3} a+\sin \sqrt{7} a|) \\
& \underbrace{+\sin \sqrt{7}((k-1) a+|\sin \sqrt{3} a+\sin \sqrt{7} a|) \mid}_{k \text { terms }}) \\
& +\sin \sqrt{7}(k a+|\sin \sqrt{3} a+\sin \sqrt{7} a| \\
& +\mid \sin \sqrt{3}(2 a+|\sin \sqrt{3} a+\sin \sqrt{7} a|) \\
& +\sin \sqrt{7}(2 a+|\sin \sqrt{3} a+\sin \sqrt{7} a|) \mid \\
& +\ldots+\mid \sin \sqrt{3}((k-1) a+|\sin \sqrt{3} a+\sin \sqrt{7} a|) \\
& \underbrace{+\sin \sqrt{7}((k-1) a+|\sin \sqrt{3} a+\sin \sqrt{7} a|) \mid}_{k \text { terms }}) \mid \text {. }
\end{aligned}
$$

Then we have

$$
\sigma(t)= \begin{cases}t, & \text { if } t \in \cup_{m=1}^{\infty}\left[p_{m}, a+p_{m}\right), \\ t+|\sin \sqrt{3} t+\sin \sqrt{7} t|, & \text { if } t \in \cup_{m=1}^{\infty}\left\{a+p_{m}\right\}\end{cases}
$$

and

$$
\mu(t)= \begin{cases}0, & \text { if } t \in \cup_{m=1}^{\infty}\left[p_{m}, a+p_{m}\right), \\ |\sin \sqrt{3} t+\sin \sqrt{7} t|, & \text { if } t \in \cup_{m=1}^{\infty}\left\{a+p_{m}\right\} .\end{cases}
$$

The concept from [12] does not include the time scale (2.5).

Remark 2.5. From Theorem 2.1, we know Definition 9 from [12] is an oriented-direction periodic time scale. Note if $\mathbb{T}$ and $\mathbb{T}_{\tau}$ approximate each other, then this approach is not an approximation between $\mathbb{T}$ and the translation of $\mathbb{T}$, but it is an approximation that occurs between $\mathbb{T}$ and a subset of $\mathbb{T}$. Note the idea in Definition 9 from [12] does not reflect approximation behavior between a time scale and its corresponding translation. We use the distance $d\left(\mathbb{T}, \mathbb{T}^{\tau}\right)$ from [6] to describe an approximation that occurs between $\mathbb{T}$ and its corresponding translation $\mathbb{T}^{\tau}$ (see Figure 1 and Figure 2). 


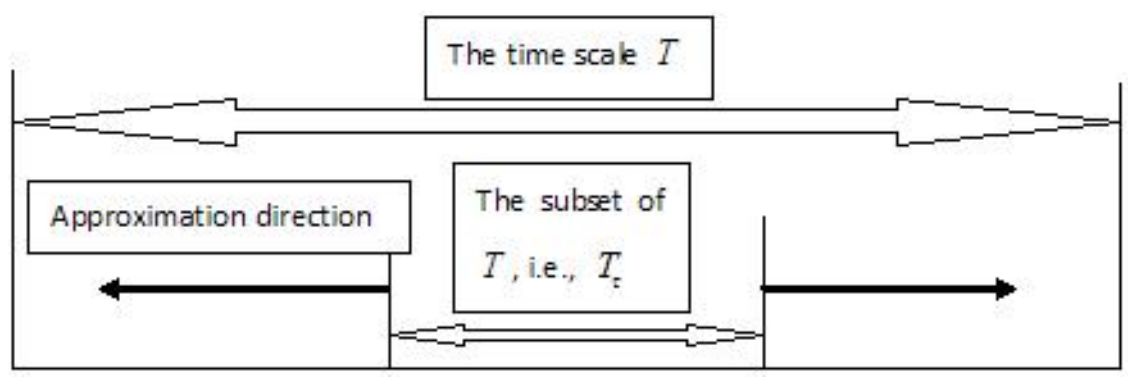

Figure 1: The approximation from [12] that will lead to the oriented-direction periodicity for the time scale $\mathbb{T}$.

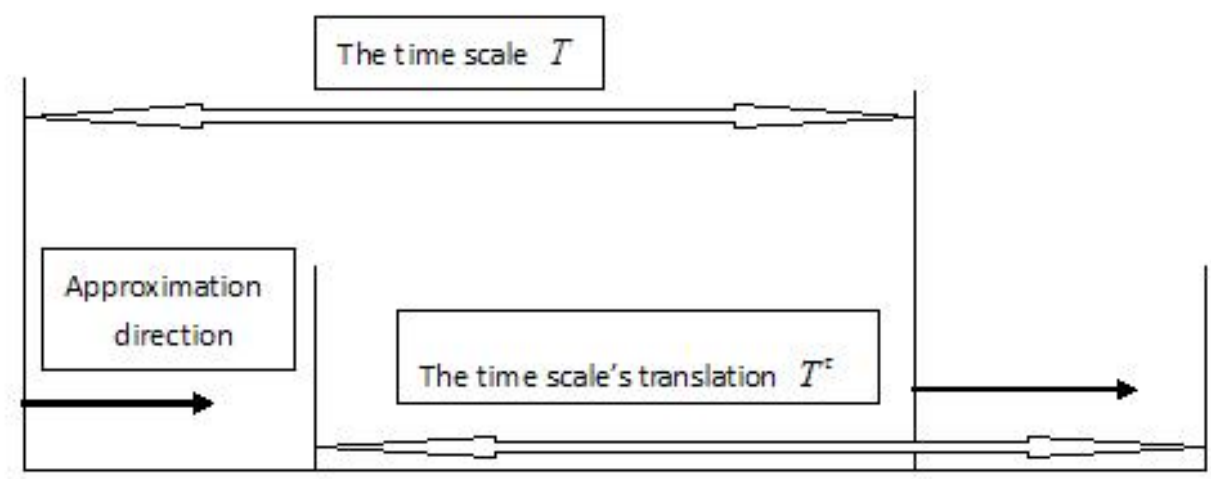

Figure 2: The approximation that describes the distance between the time scale $\mathbb{T}$ and its translation $\mathbb{T}^{\tau}$ from [6].

Remark 2.6. We can use the similar idea of Definition 2.3 and [9] to introduce a new concept of periodic time scales in shift operators (i.e., Definition 2.4).

Definition 2.4. Let $\delta: \mathbb{R} \times \mathbb{T} \rightarrow \mathbb{T}$ be a shift operator associated with the initial point $t_{0}$, so $\delta\left(t_{0}, t\right)=t$. Denote

$$
\mathbb{T}^{\delta_{\tau}}:=\delta(\tau, \mathbb{T})=\{\delta(\tau, t), \forall t \in \mathbb{T}\},
$$

we say $\mathbb{T}$ is a periodic time scale under the shift operator $\delta$ if

$$
\Pi_{\delta}:=\left\{\tau \in \mathbb{R}: \mathbb{T}^{\delta_{\tau}} \subseteq \mathbb{T}\right\} \neq\left\{t_{0}\right\} .
$$

Furthermore, denote

$$
\Pi_{\delta_{ \pm}}:=\left\{\tau \in \mathbb{R}: \delta_{ \pm}(\tau, t) \in \mathbb{T}, \forall t \in \mathbb{T}\right\},
$$

if $\Pi_{\delta_{+}} \neq\left\{t_{0}\right\}$, we say $\mathbb{T}$ is a positive-direction periodic time scale under the shift operator $\delta_{+}$; if $\Pi_{\delta_{-}} \neq\left\{t_{0}\right\}$, we say $\mathbb{T}$ is a negative-direction periodic time scale under the shift operator $\delta_{-}$; if $\Pi_{\delta_{+}} \neq\left\{t_{0}\right\}$, we say $\mathbb{T}$ is a bidirection periodic time scale under the shift operators $\delta_{ \pm}$, where the shift operators $\delta_{ \pm}$satisfy all the conditions from [9].

Note that under the above Definition 2.4, the time scale (1.2) can be regarded as a positive-direction periodic time scale under the shift operator $\delta_{+}$since $\Pi_{\delta_{+}} \neq\left\{t_{0}\right\}=\{1\}$, but (1.2) is not a bi-direction periodic time scale under the shift operator $\delta_{ \pm}$since for the shift operator $\delta_{+}$, there not exists the corresponding shift operator $\delta_{-}$for this time scale. Hence, the new concept of periodic time scales from [9] is still a bi-direction periodic time scale under the shift operators, it is a particular case of Definition 2.4.

In what follows, we emphasize that the time scales distance is measured by the Hausdorff distance. 
Definition 2.5 ([13]). Let $X$ and $Y$ be two non-empty subsets of a metric space $(M, d)$. We define their Hausdorff distance $d(X, Y)$ by

$$
d(X, Y)=\max \left\{\sup _{x \in X} \inf _{y \in Y} \tilde{d}(x, y), \sup _{y \in Y} \inf _{x \in X} \tilde{d}(x, y)\right\},
$$

where $\tilde{d}(\cdot, \cdot)$ denotes the distance between two points (see Figure 3 ).

Hence, from Definition 2.5, if we assume that $X=\mathbb{T}_{1}$ and $Y=\mathbb{T}_{2}$, this well-known definition is

$$
d\left(\mathbb{T}_{1}, \mathbb{T}_{2}\right)=\max \left\{\sup _{t \in \mathbb{T}_{1}} \inf _{s \in \mathbb{T}_{2}} \tilde{d}(t, s), \sup _{s \in \mathbb{T}_{2}} \inf _{t \in \mathbb{T}_{1}} \tilde{d}(t, s)\right\},
$$

which is Definition 7 in [12]. In [6], the authors let $\tau$ be a number and set the time scales:

$$
\mathbb{T}:=\bigcup_{i=-\infty}^{+\infty}\left[\alpha_{i}, \beta_{i}\right], \quad \mathbb{T}^{\tau}:=\mathbb{T}+\tau=\{t+\tau: \forall t \in \mathbb{T}\}:=\bigcup_{i=-\infty}^{+\infty}\left[\alpha_{i}^{\tau}, \beta_{i}^{\tau}\right] .
$$

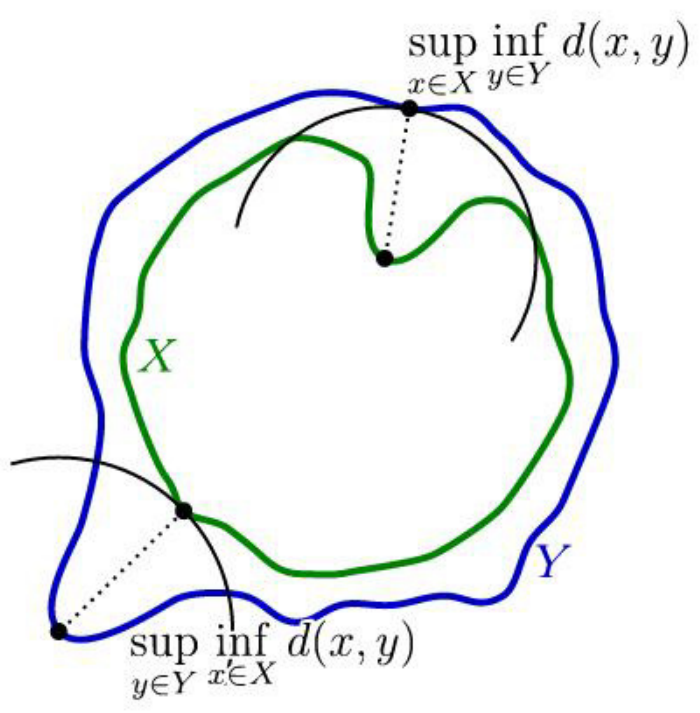

Figure 3: Components of the calculation of the Hausdorff distance between the green line $X$ and the blue line $Y$.

Define the distance between two time scales, $\mathbb{T}$ and $\mathbb{T}^{\tau}$ by

$$
d\left(\mathbb{T}, \mathbb{T}^{\tau}\right)=\max \left\{\sup _{i \in \mathbb{Z}}\left|\alpha_{i}-\alpha_{i}^{\tau}\right|, \sup _{i \in \mathbb{Z}}\left|\beta_{i}-\beta_{i}^{\tau}\right|\right\},
$$

where

$$
\alpha_{i}^{\tau}:=\inf \left\{\alpha \in \mathbb{T}^{\tau}:\left|\alpha_{i}-\alpha\right|\right\} \text { and } \beta_{i}^{\tau}:=\inf \left\{\beta \in \mathbb{T}^{\tau}:\left|\beta_{i}-\beta\right|\right\} .
$$

Note if we let $X=\mathbb{T}$ and $Y=\mathbb{T}^{\tau}$ in Definition 2.5, then (2.6) immediately turns into (2.8) and we can calculate the distance between $\mathbb{T}$ and $\mathbb{T}^{\tau}$ from the distance of their intervals, i.e, the formula (2.8) (see Figure 4).

Now we introduce some notations to make the definitions clearer in [6].

Definition 2.6. Let $\mathbb{T}$ be an oriented-direction intersection time scale. We say $\mathbb{T}$ is an almost periodic time scale if for any given $\varepsilon>0$, there exists a constant $l(\varepsilon)>0$ such that each interval of length $l(\varepsilon)$ contains a $\tau(\varepsilon) \in \Pi_{1}$ such that

$$
d\left(\mathbb{T}, \mathbb{T}^{\tau}\right)<\varepsilon,
$$




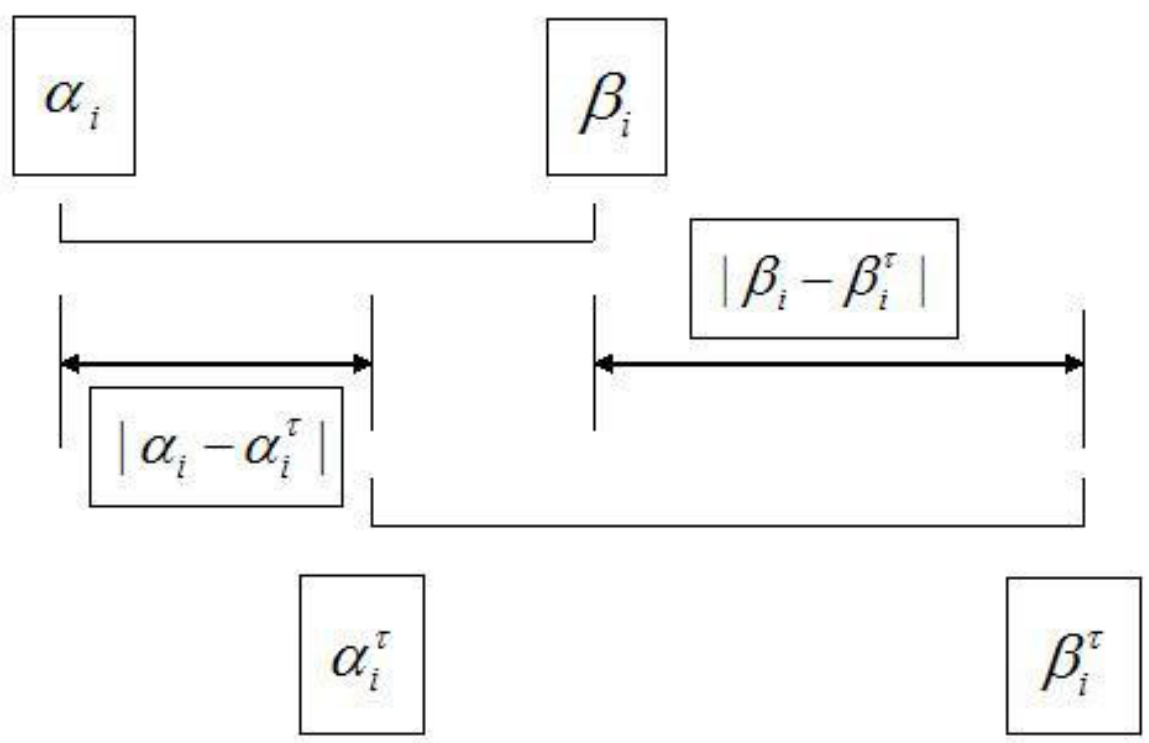

Figure 4: The Hausdorff distance between the interval $\left[\alpha_{i}, \beta_{i}\right]$ and the interval $\left[\alpha_{i}^{\tau}, \beta_{i}^{\tau}\right]$.

i.e., for any $\varepsilon>0$, the following set

$$
E\{\mathbb{T}, \varepsilon\}=\left\{\tau \in \Pi_{1}: d\left(\mathbb{T}^{\tau}, \mathbb{T}\right)<\varepsilon\right\}
$$

is relatively dense in $\Pi_{1}$. Here $\tau$ is called the $\varepsilon$-translation number of $\mathbb{T}$ and $l(\varepsilon)$ is called the inclusion length of $E\{\mathbb{T}, \varepsilon\}, E\{\mathbb{T}, \varepsilon\}$ is called the $\varepsilon$-translation set of $\mathbb{T}$, and for simplicity, we use the notation $E\{\mathbb{T}, \varepsilon\}:=\Pi_{\varepsilon}$.

Remark 2.7. From the property of the set $\Pi_{1}$, we no longer worry about the case $\mathbb{T} \cap \mathbb{T}^{\tau}=\emptyset$ in Definition 2.6. We observe that such a revision does not have any impact on the results in [6].

Remark 2.8. According to Definition 2.6, if $\mathbb{T}$ is a bi-direction intersection time scale, then one can obtain that $\sup \mathbb{T}=+\infty, \inf \mathbb{T}=-\infty$ and

$$
\sup \left\{\mathbb{T} \cap \mathbb{T}^{\tau}\right\}=+\infty, \quad \inf \left\{\mathbb{T} \cap \mathbb{T}^{\tau}\right\}=-\infty
$$

Furthermore, for a bi-direction intersection time scale, one can see that if $d\left(\mathbb{T}, \mathbb{T}^{\tau}\right)<\varepsilon$, then $d\left(\mathbb{T}, \mathbb{T}^{-\tau}\right)<\varepsilon$, i.e., if $\tau \in E\{\mathbb{T}, \varepsilon\}$, then $-\tau \in E\{\mathbb{T}, \varepsilon\}$. If $\tau_{1} \in E\{\mathbb{T}, \varepsilon\}, \tau_{2} \in E\{\mathbb{T}, \varepsilon\}$, then we have $\tau_{1}+\tau_{2} \in E\{\mathbb{T}, 2 \varepsilon\}$ since

$$
d\left(\mathbb{T}, \mathbb{T}^{\tau_{1}+\tau_{2}}\right) \leq d\left(\mathbb{T}, \mathbb{T}^{\tau_{1}}\right)+d\left(\mathbb{T}^{\tau_{1}}, \mathbb{T}^{\tau_{1}+\tau_{2}}\right)=d\left(\mathbb{T}, \mathbb{T}^{\tau_{1}}\right)+d\left(\mathbb{T}, \mathbb{T}^{\tau_{2}}\right)<2 \varepsilon .
$$

Remark 2.9. In Definition 2.6, if for any $p>0, \tau \in E\{\mathbb{T}, \varepsilon\} \cap(p,+\infty)$, then we say $\mathbb{T}$ is a positive-direction almost periodic time scale; if for any $q<0, \tau \in E\{\mathbb{T}, \varepsilon\} \cap(-\infty, q)$, then we say $\mathbb{T}$ is a negative-direction almost periodic time scale; if for any $p>0, q<0, \pm \tau \in E\{\mathbb{T}, \varepsilon\} \cap[(-\infty, q) \cup(p,+\infty)]$, then we say $\mathbb{T}$ is a bi-direction almost periodic time scale.

From Definition 2.6, we note the following theorems.

Theorem 2.2 ([6]). Let $\mathbb{T}$ be an almost periodic time scale. Then for any given sequence $\alpha^{\prime} \subset \Pi_{1}$, there exists a subsequence $\alpha \subset \alpha^{\prime}$ such that $\left\{\mathbb{T}^{\alpha^{n}}\right\}$ converges to a time scale $\mathbb{T}_{0}$, i.e., for any given $\varepsilon>0$, there exists $N_{0}>0$ such that $n>N_{0}$ implies $d\left(\mathbb{T}^{\alpha_{n}}, \mathbb{T}_{0}\right)<\varepsilon$. Furthermore, $\mathbb{T}_{0}$ is also almost periodic. 
Theorem 2.3 ([6]). Let $\mathbb{T}$ be a time scale. If for any sequence $\alpha^{\prime} \subset \Pi_{1}$, there exists $\alpha \subset \alpha^{\prime}$ such that $\left\{\mathbb{T}^{\alpha_{n}}\right\}$ converges to a time scale $\mathbb{T}_{0}$, then $\mathbb{T}$ is almost periodic.

From Theorems 2.2 and 2.3, we can obtain the following equivalent definition of almost periodic time scales.

Definition 2.7 ([6]). Let $\mathbb{T}$ be a time scale. If for any given sequence $\alpha^{\prime} \subset \Pi_{1}$, there exists a subsequence $\alpha \subset \alpha^{\prime}$ such that $\left\{\mathbb{T}^{\alpha_{n}}\right\}$ converges to a time scale $\mathbb{T}_{0}$, then $\mathbb{T}$ is called an almost periodic time scale.

Remark 2.10. From Theorems 2.2-2.3 we note from Theorem 2.1, that the results in [6] are valid for an orienteddirection periodic time scale.

Based on Definitions 2.6 and 2.7, we give two equivalent concepts of almost periodic functions on time scales. We shall use the following notation: $\mathbb{E}^{n}$ denotes $\mathbb{R}^{n}$ or $\mathbb{C}^{n}, D$ denotes an open set in $\mathbb{E}^{n}$ or $D=\mathbb{E}^{n}$, and $S$ denotes an arbitrary compact subset of $D$.

Definition 2.8. Let $\mathbb{T}$ be an almost periodic time scale, i.e., $\mathbb{T}$ satisfies Definition 2.6. A function $f \in C\left(\mathbb{T} \times D, \mathbb{E}^{n}\right)$ is called an almost periodic function in $t \in \mathbb{T}$ uniformly for $x \in D$ if the $\varepsilon_{2}$-translation set of $f$

$$
E\left\{\varepsilon_{2}, f, S\right\}=\left\{\tau \in \Pi_{\varepsilon_{1}}:|f(t+\tau, x)-f(t, x)|<\varepsilon_{2}, \text { for all }(t, x) \in\left(\mathbb{T} \cap \mathbb{T}^{-\tau}\right) \times S\right\}
$$

is a relatively dense set in $\Pi_{\varepsilon_{1}}$ for all $\varepsilon_{2}>\varepsilon_{1}>0$ and for each compact subset $S$ of $D$; that is, for any given $\varepsilon_{2}>\varepsilon_{1}>0$ and each compact subset $S$ of $D$, there exists a constant $l\left(\varepsilon_{2}, S\right)>0$ such that each interval of length $l\left(\varepsilon_{2}, S\right)$ contains a $\tau\left(\varepsilon_{2}, S\right) \in E\left\{\varepsilon_{2}, f, S\right\}$ such that

$$
|f(t+\tau, x)-f(t, x)|<\varepsilon_{2}, \quad \text { for all }(t, x) \in\left(\mathbb{T} \cap \mathbb{T}^{-\tau}\right) \times S .
$$

This $\tau$ is called the $\varepsilon_{2}$-translation number of $f$ and $l\left(\varepsilon_{2}, S\right)$ is called the inclusion length of $E\left\{\varepsilon_{2}, f, S\right\}$.

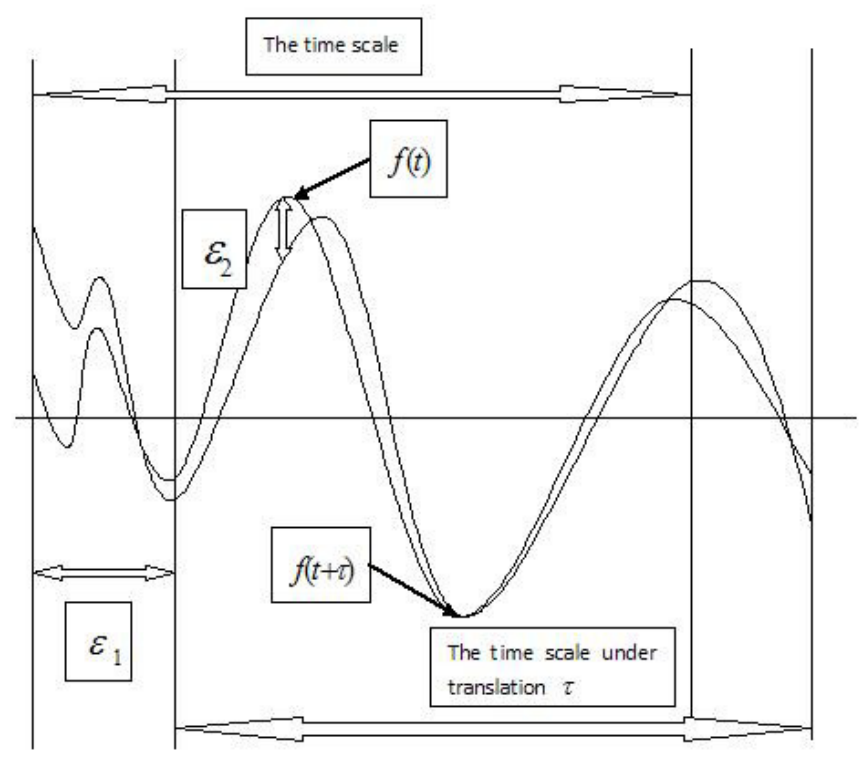

Figure 5: The functions approximate each other along with the approximation of their respective time scales. 
Remark 2.11. The positive number $\varepsilon_{1}$ in Definition 2.6 should be sufficiently small. Clearly, if $\varepsilon_{1}=0$, then Definition 2.8 is equivalent to Definition 1.8 in [10], i.e., the concept of almost periodic functions on periodic time scales. It is worth noting that because $\varepsilon_{2}>\varepsilon_{1}>0$, if $\varepsilon_{1}>0$ is an arbitrarily small positive number, then $\varepsilon_{2}$ can be an arbitrarily small positive number, i.e., the approximation of the translation of time scales is the prerequisite that guarantees the approximation of the translation of functions defined on time scales (see Figure 5).

Remark 2.12. Note in Definition 16 of [12], the $\varepsilon$ for the time scales equals the $\varepsilon$ for the functions, and $\varepsilon$ is an arbitrary positive number, which will lead to that if $\varepsilon=0$, then $\mathbb{T}=\mathbb{T} \cap \mathbb{T}^{-\tau}$ and $f(t)=f(t+\tau)$. Hence, in Definition 16 in [12] we have a $\tau$-periodic function on a $\tau$-periodic time scale i.e, in fact, $f(t)$ defined in Definition 16 of [12] is a periodic function on a periodic time scale in nature.

Now, let

$$
\Pi_{\varepsilon}=E\{\mathbb{T}, \varepsilon\}, \mathbb{T}^{\Pi_{\varepsilon}}=\left\{\mathbb{T}^{-\tau}:-\tau \in E\{\mathbb{T}, \varepsilon\}\right\} .
$$

Then, Definition 2.8 can be stated as follows:

Definition 2.9 ([4]). Let $\mathbb{T}$ be an almost periodic time scale. A function $f \in C\left(\mathbb{T} \times D, \mathbb{E}^{n}\right)$ is called an almost periodic function in $t \in \mathbb{T}$ uniformly for $x \in D$ if the $\varepsilon_{2}$-translation set of $f$

$$
\begin{aligned}
E\left\{\varepsilon_{2}, f, S\right\}= & \left\{\tau \in \Pi_{\mathcal{\varepsilon}_{1}}:|f(t+\tau, x)-f(t, x)|<\varepsilon_{2},\right. \\
& \text { for all } \left.(t, x) \in\left(\mathbb{T} \cap\left(\cup \mathbb{T}^{\Pi_{\varepsilon_{1}}}\right)\right) \times S\right\}
\end{aligned}
$$

is a relatively dense set in $\Pi_{\varepsilon_{1}}$ for all $\varepsilon_{2}>\varepsilon_{1}>0$ and for each compact subset $S$ of $D$; that is, for any given $\varepsilon_{2}>\varepsilon_{1}>0$ and each compact subset $S$ of $D$, there exists a constant $l\left(\varepsilon_{2}, S\right)>0$ such that each interval of length $l\left(\varepsilon_{2}, S\right)$ contains a $\tau\left(\varepsilon_{2}, S\right) \in E\left\{\varepsilon_{2}, f, S\right\}$ such that

$$
|f(t+\tau, x)-f(t, x)|<\varepsilon_{2}, \quad \text { for all }(t, x) \in\left(\mathbb{T} \cap\left(\cup \mathbb{T}^{\Pi_{\varepsilon_{1}}}\right)\right) \times S .
$$

Here $\tau$ is called the $\varepsilon_{2}$-translation number of $f$ and $l\left(\varepsilon_{2}, S\right)$ is called the inclusion length of $E\left\{\varepsilon_{2}, f, S\right\}$.

Based on Definitions 2.6 and 2.8, we will give the second equivalent concept of almost periodic functions on time scales:

Definition 2.10. Assume that $\mathbb{T}$ is an almost periodic time scale. Let $f(t, x) \in C\left(\mathbb{T} \times D\right.$, $\left.\mathbb{E}^{n}\right)$. If for any given sequence $\alpha^{\prime} \subset \Pi_{1}$, there exists a subsequence $\alpha \subset \alpha^{\prime}$ such that the limit set $\mathbb{T}_{0}$ of $\left\{\mathbb{T}^{-\alpha_{n}}\right\}$ exists and $T_{\alpha} f(t, x)$ exists uniformly on $\mathbb{T}_{0} \times S$, then $f(t, x)$ is called an almost periodic function in $t$ uniformly for $x \in D$.

Lemma 2.2. If $\mathbb{T}$ is an almost periodic time scale, then for any $\varepsilon>0$ and any right dense point $t \in \mathbb{T}$, there exists a constant $l(\varepsilon)>0$ such that each interval of length $l(\varepsilon)$ contains a $\tau(\varepsilon) \in \Pi_{1}$ such that there exists a sequence $\left\{t_{n}\right\} \subseteq \mathbb{T} \cap \mathbb{T}^{\tau}$ and $\lim _{n \rightarrow \infty} t_{n}=t, t_{n}>t, n \in \mathbb{N}$.

Proof. Since $\mathbb{T}$ is an almost periodic time scale, then for any $\varepsilon>0$, there exists a constant $l(\varepsilon)>0$ such that each interval of length $l(\varepsilon)$ contains a $\tau(\varepsilon) \in \Pi$ such that $d\left(\mathbb{T}, \mathbb{T}^{\tau}\right)<\varepsilon$. Because $t \in \mathbb{T}$ is a right dense point in $\mathbb{T}$, then there exists a sequence $\left\{t_{n}\right\} \subseteq \mathbb{T}$ and $t_{n}>t, n \in \mathbb{N}$ such that $\lim _{n \rightarrow \infty} t_{n}=t$. We argue by contradiction. If $\left\{t_{n}\right\} / \subset \mathbb{T}^{\tau}$, then $t / \in \mathbb{T}^{\tau}$, which implies that there exists some $t^{\star} \in \mathbb{T}^{\tau}$ such that $d\left(\mathbb{T}, \mathbb{T}^{\tau}\right) \geq\left|t-t^{\star}\right|>0$, and this is a contradiction. Hence, we have $\left\{t_{n}\right\} \subseteq \mathbb{T}^{\tau}$. Therefore, there exists a sequence $\left\{t_{n}\right\} \subseteq \mathbb{T} \cap \mathbb{T}^{\tau}$ and $\lim _{n \rightarrow \infty} t_{n}=t, t_{n}>t, n \in \mathbb{N}$. This completes the proof.

Theorem 2.4. If $\mathbb{T}$ is an almost periodic time scale, then for any $\varepsilon>0$, there exists a constant $l(\varepsilon)>0$ such that each interval of length $l(\varepsilon)$ contains a $\tau(\varepsilon) \in E\{\varepsilon, \mu\}$ such that

$$
|\mu(t+\tau)-\mu(t)|<\varepsilon, \quad \text { for all } t \in \mathbb{T} \cap \mathbb{T}^{-\tau} .
$$




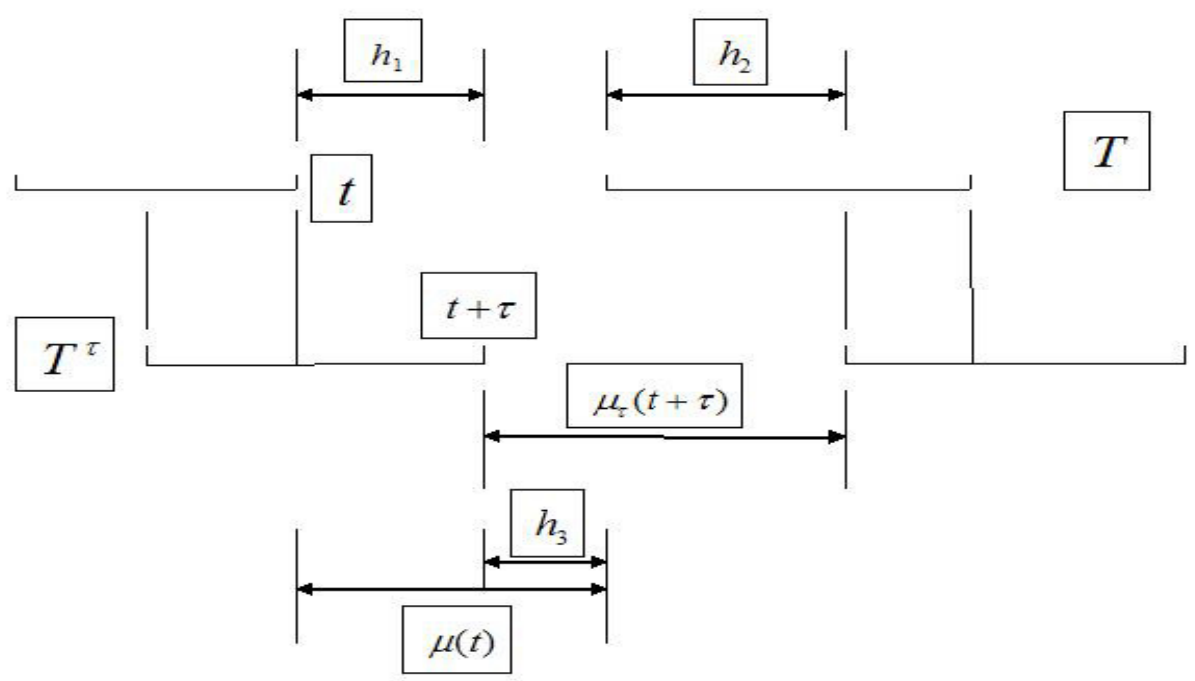

Figure 6: The relationship between $d\left(\mathbb{T}, \mathbb{T}^{\tau}\right)$ and the graininess function $\mu$.

Proof. First, let $\mathbb{T}$ be an almost periodic time scale. We claim that: for any $\tau \in \Pi_{\varepsilon}$, if $t$ is a right scattered point in $\mathbb{T}$, then $t+\tau$ are right scattered points in $\mathbb{T}$; if $t$ is a right dense point in $\mathbb{T}$, then $t+\tau$ are right dense points in $\mathbb{T}$.

In fact, if $t \in \mathbb{T} \cap \mathbb{T}^{-\tau}$ is a right scattered point in $\mathbb{T}$, then $t+\tau$ are right scattered points in $\mathbb{T}$. We argue by contradiction. Assume that $t+\tau \in \mathbb{T}$ is a right dense point in $\mathbb{T}$, then by Lemma 2.2, there exists a sequence $\left\{t_{n}\right\} \subseteq\left(\mathbb{T} \cap \mathbb{T}^{\tau}\right)$ and $t_{n} \geq t+\tau, n \in \mathbb{N}$ such that $\lim _{n \rightarrow \infty} t_{n}=t+\tau$. Hence, we have $\lim _{n \rightarrow \infty} t_{n}-\tau=t$, i.e., $\left\{t_{n}-\tau\right\} \subseteq\left(\mathbb{T} \cap \mathbb{T}^{-\tau}\right) \subseteq \mathbb{T}$. So $t$ is a right dense point in $\mathbb{T}$, which is a contradiction. Thus, $t+\tau$ is a right scattered point in $\mathbb{T}$.

On the other hand, if $t \in \mathbb{T} \cap \mathbb{T}^{-\tau}$ is a right dense point in $\mathbb{T}$, then $t+\tau$ are right dense points in $\mathbb{T}$. In fact, by Lemma 2.2, there exists a sequence $\left\{t_{n}\right\} \subseteq \mathbb{T} \cap \mathbb{T}^{-\tau}$ and $t_{n} \geq t, n \in \mathbb{N}$ such that $\lim _{n \rightarrow \infty} t_{n}=t$. Then we have $\lim _{n \rightarrow \infty} t_{n}+\tau=t+\tau$ and $\left\{t_{n}+\tau\right\} \subseteq \mathbb{T} \cap \mathbb{T}^{\tau} \subset \mathbb{T}$. Thus, $t+\tau$ is a right dense point in $\mathbb{T}$.

Hence, $t \in \mathbb{T} \cap \mathbb{T}^{-\tau}$ implies that $t, t+\tau \in \mathbb{T}$. When $t$ is a right dense point, then $t+\tau$ is also a right dense point, so we have $\mu(t)=\mu(t+\tau)=0$. When $t$ is a right scattered point, then $t+\tau$ is also a right scattered point and

$$
|\mu(t)-\mu(t+\tau)| \leq d\left(\mathbb{T}, \mathbb{T}^{\tau}\right)<\varepsilon
$$

This completes the proof.

Remark 2.13. From Figure 6, let $\mathbb{T}$ be an almost periodic time scale and $\mu_{\tau}: \mathbb{T}^{\tau} \rightarrow \mathbb{R}^{+}$be the graininess function on $\mathbb{T}^{\tau}$, and then we obtain that

$$
\begin{aligned}
\left|\mu(t)-\mu_{\tau}(t+\tau)\right| & \leq \max \left\{\left|\mu_{\tau}(t+\tau)-h_{3}\right|,\left|\mu(t)-h_{3}\right|\right\} \\
& =\max \left\{h_{2}, h_{1}\right\} \leq d\left(\mathbb{T}, \mathbb{T}^{\tau}\right)<\varepsilon \text { for all } t \in \mathbb{T} .
\end{aligned}
$$

Particularly, if $t \in\left(\mathbb{T} \cap \mathbb{T}^{-\tau}\right) \subset \mathbb{T}$, then $t+\tau \in \mathbb{T}$ and $\mu_{\tau}(t+\tau)=\mu(t+\tau)$. Hence, from (2.11), we obtain (2.10). Furthermore, if $t+\tau / \in \mathbb{T}$, i.e., $t / \in \mathbb{T} \cap \mathbb{T}^{-\tau}$, we get $\mu_{\tau}(t+\tau)=\mu(t)$. 
Remark 2.14. The inequality (2.10) can also be written as

$$
|\sigma(t+\tau)-\sigma(t)-\tau|<\varepsilon, \quad \text { for all } t \in \mathbb{T} \cap \mathbb{T}^{-\tau},
$$

which indicates that if $\mathbb{T}$ is $\tau$-periodic, then $\sigma(t+\tau)=\sigma(t)+\tau$.

Now, from Definition 2.6 and the Hausdorff distance (2.8), we can give the third concept of almost periodic time scales by the graininess function $\mu$ as follows:

Definition 2.11. Let $\mu: \mathbb{T} \rightarrow \mathbb{R}^{+}, \mu(t)=\sigma(t)-t$ and $\mu_{\tau}: \mathbb{T}^{\tau} \rightarrow \mathbb{R}^{+}$. We say $\mathbb{T}$ is an almost periodic time scale if for any $\varepsilon>0$, the set

$$
\Pi^{\star}=\left\{\tau \in \Pi_{1}:\left|\mu_{\tau}(t+\tau)-\mu(t)\right|<\varepsilon, \forall t \in \mathbb{T}\right\}
$$

is relatively dense in $\Pi_{1}$.

Remark 2.15. Note that Definition 2.11 implies the following:

(1) If $t \in\left(\mathbb{T} \cap \mathbb{T}^{-\tau}\right) \subset \mathbb{T}$, then $t+\tau \in \mathbb{T}$ and $\mu_{\tau}(t+\tau)=\mu(t+\tau)$. Hence, from (2.12), we obtain (2.10). Furthermore, if $t \in \mathbb{T} \cap \mathbb{T}^{-\tau}$ is a right dense point in $\mathbb{T}$, it follows from (2.12) that $t+\tau \in \mathbb{T}$ is also a right dense point. Similarly, if $t \in \mathbb{T} \cap \mathbb{T}^{-\tau}$ is a right scattered point in $\mathbb{T}$, by (2.12), then $t+\tau \in \mathbb{T}$ is also a right scattered point. In fact, if $t \in \mathbb{T} \cap \mathbb{T}^{-\tau}$ is a right dense point in $\mathbb{T}$, then $\mu(t)=0$. From (2.12), we know that $\mu(t+\tau)=0$, so $t+\tau \in \mathbb{T}$ is a right dense point. Similarly, if $t \in \mathbb{T} \cap \mathbb{T}^{-\tau}$ is a right scattered point, then $\mu(t)>0$. From (2.12), we have

$$
\mu(t+\tau)=|\mu(t)-\mu(t)+\mu(t+\tau)| \geq|| \mu(t)|-| \mu(t+\tau)-\mu(t)|| \geq \mu(t)-\varepsilon>0 .
$$

Hence, $t+\tau$ is a right scattered point.

(2) From Figure 6 and (2.12), we obtain that

$$
d\left(\mathbb{T}, \mathbb{T}^{\tau}\right) \leq \sup _{t \in \mathbb{T}}\left|\mu_{\tau}(t+\tau)-\mu(t)\right|<\varepsilon,
$$

which indicates that $\mathbb{T}$ is an oriented-direction almost periodic time scale.

(3) From Figure 6 and Remark 2.13, $\mathbb{T}^{\tau}$ is a $\tau$-translation of $\mathbb{T}$. Let $\mu_{\tau}: \mathbb{T}^{\tau} \rightarrow \mathbb{R}^{+}$be the graininess function of $\mathbb{T}^{\tau}$, and we obtain that

$$
\mu_{\tau}(t+\tau)= \begin{cases}\mu(t), & t+\tau / \in \mathbb{T}, \\ \mu(t+\tau), & t+\tau \in \mathbb{T} .\end{cases}
$$

Thus, from (2.13), we can simplify Definition 2.11 as follows:

Let $\mu: \mathbb{T} \rightarrow \mathbb{R}^{+}$be a graininess function of $\mathbb{T}$. We say $\mathbb{T}$ is an almost periodic time scale if for any $\varepsilon>0$, the set

$$
\Pi^{\star}=\left\{\tau \in \Pi_{1}:|\mu(t+\tau)-\mu(t)|<\varepsilon, \forall t \in \mathbb{T} \cap \mathbb{T}^{-\tau}\right\}
$$

is relatively dense in $\Pi_{1}$.

Remark 2.16. From Example 2.1, we know $\mu(t)$ has almost periodicity at all its right scattered points. Clearly, $\mu(t)$ satisfies all the conditions in Definition 2.11, and thus, the time scale $\mathbb{T}$ from Example 2.1 is an almost periodic time scale. Although $|\inf \mathbb{T}|<+\infty$, (1.1) and Example 2.1 can be regarded as a periodic time scale and an almost periodic time scale with sup $\mathbb{T}=+\infty$, respectively (i.e., they are positive-direction translation time scales).

\section{Changing-periodic time scales}

In [2], Wang and Agarwal proposed a new concept called changing-periodic time scales. Now, in this section, considering Definition 2.3, we will improve and obtain some new results about changing-periodic time scales. 
Definition 3.1 ([2]). Let $\mathbb{T}$ be an infinite time scale. We say $\mathbb{T}$ is a changing-periodic or a piecewise-periodic time scale if the following conditions are fulfilled:

(a) $\mathbb{T}=\left(\bigcup_{i=1}^{\infty} \mathbb{T}_{i}\right) \cup \mathbb{T}_{r}$ and $\left\{\mathbb{T}_{i}\right\}_{i \in \mathbb{Z}^{+}}$is a well connected timescale sequence, where $\mathbb{T}_{r}=\bigcup_{i=1}^{k}\left[\alpha_{i}, \beta_{i}\right]$ and $k$ is some finite number, and $\left[\alpha_{i}, \beta_{i}\right]$ are closed intervals for $i=1,2, \ldots, k$ or $\mathbb{T}_{r}=\emptyset$;

(b) $S_{i}$ is a nonempty subsets of $\mathbb{R}$ with $0 / \in S_{i}$ for each $i \in \mathbb{Z}^{+}$and $\Pi=\left(\bigcup_{i=1}^{\infty} S_{i}\right) \cup R_{0}$, where $R_{0}=\{0\}$ or $R_{0}=\emptyset$;

(c) for all $t \in \mathbb{T}_{i}$ and all $\omega \in S_{i}$, we have $t+\omega \in \mathbb{T}_{i}$, i.e., $\mathbb{T}_{i}$ is an $\omega$-periodic time scale;

(d) for $i \neq j$, for all $t \in \mathbb{T}_{i} \backslash\left\{t_{i j}^{k}\right\}$ and all $\omega \in S_{j}$, we have $t+\omega / \in \mathbb{T}$, where $\left\{t_{i j}^{k}\right\}$ is the connected points set of the timescale sequence $\left\{\mathbb{T}_{i}\right\}_{i \in \mathbb{Z}^{+}}$;

(e) $R_{0}=\{0\}$ if and only if $\mathbb{T}_{r}$ is a zero-periodic time scale and $R_{0}=\emptyset$ if and only if $\mathbb{T}_{r}=\emptyset$;

and the set $\Pi$ is called a changing-periods set of $\mathbb{T}, \mathbb{T}_{i}$ is called the periodic sub-timescale of $\mathbb{T}$ and $S_{i}$ is called the periods subset of $\mathbb{T}$ or the periods set of $\mathbb{T}_{i}, \mathbb{T}_{r}$ is called the remain timescale of $\mathbb{T}$ and $R_{0}$ the remain periods set of $\mathbb{T}$.

Remark 3.1. Note that from condition (c) in Definition 3.1, we should know that $\mathbb{T}_{i}$ is an oriented-direction periodic time scale. If $\mathbb{T}$ is a bi-direction periodic time scale, then it is Definition 3.7 in [8]. We adopt Definition 2.3 to understand it. Now, (2.1) can be written as

$$
\Pi_{2}=\{\tau \in \mathbb{R}: t+\tau \in \mathbb{T}, \forall t \in \mathbb{T}\} \neq\{0\},
$$

rather than

$$
\Pi_{2}=\{\tau \in \mathbb{R}: t \pm \tau \in \mathbb{T}, \forall t \in \mathbb{T}\} \neq\{0\},
$$

since (3.2) is a bi-direction periodic time scale and $\sup \mathbb{T}=+\infty, \inf \mathbb{T}=-\infty$, and it is quite limited for understanding the decomposition theorem of time scales. From condition (c) in Definition 3.1, we see that $\mathbb{T}_{i}$ is a periodic time scale implies $\mathbb{T}_{i}$ satisfies (3.1) rather than (3.2), i.e., $\mathbb{T}_{i}$ is an oriented-direction periodic time scale.

Example 3.1. Let $k \in \mathbb{Z}$, and consider the following time scale:

$$
\mathbb{T}=\left\{\bigcup_{k=-\infty}^{+\infty}\left[\frac{3}{2}(2 k+1), \frac{3}{2}(2 k+1)+\frac{1}{12}\right]\right\} \bigcup\left\{\bigcup_{k=-\infty}^{+\infty}\left[\frac{3 \sqrt{2}}{2}(2 k+1), \frac{3 \sqrt{2}}{2}(2 k+1)+\frac{\sqrt{3}}{5}\right]\right\} .
$$

We denote

$$
\mathbb{T}_{1}=\bigcup_{k=-\infty}^{+\infty}\left[\frac{3}{2}(2 k+1), \frac{3}{2}(2 k+1)+\frac{1}{12}\right] \text { and } \mathbb{T}_{2}=\bigcup_{k=-\infty}^{+\infty}\left[\frac{3 \sqrt{2}}{2}(2 k+1), \frac{3 \sqrt{2}}{2}(2 k+1)+\frac{\sqrt{3}}{5}\right] .
$$

Then, by a direct calculation the set $\Pi_{2}$ is

$$
\Pi_{2}=\{3 n, n \in \mathbb{Z}\} \bigcup\{3 \sqrt{2} n, n \in \mathbb{Z}\}:=S_{1} \cup S_{2} .
$$

This time scale is a changing-periodic time scale according to Definition 3.1.

Example 3.2. Let $k \in \mathbb{Z}$, and consider the following time scale:

$$
\mathbb{T}=\left\{\bigcup_{k=0}^{+\infty}\left[-\frac{3}{2}(2 k+1),-\frac{3}{2}(2 k+1)-\frac{1}{12}\right]\right\} \bigcup\left\{\bigcup_{k=0}^{+\infty}\left[\frac{3 \sqrt{2}}{2}(2 k+1), \frac{3 \sqrt{2}}{2}(2 k+1)+\frac{\sqrt{3}}{5}\right]\right\}
$$

We denote

$$
\mathbb{T}_{1}=\bigcup_{k=0}^{+\infty}\left[-\frac{3}{2}(2 k+1),-\frac{3}{2}(2 k+1)-\frac{1}{12}\right] \text { and } \mathbb{T}_{2}=\bigcup_{k=0}^{+\infty}\left[\frac{3 \sqrt{2}}{2}(2 k+1), \frac{3 \sqrt{2}}{2}(2 k+1)+\frac{\sqrt{3}}{5}\right]
$$


Then, by direct calculation the set $\Pi_{2}$ is

$$
\Pi_{2}=\{-3 n, n \in \mathbb{N}\} \bigcup\{3 \sqrt{2} n, n \in \mathbb{N}\}:=S_{1} \cup S_{2} .
$$

According to Definition 3.1, this time scale is a changing-periodic time scale in which $\mathbb{T}_{1}$ is a negative-direction periodic sub-timescale and $\mathbb{T}_{2}$ is a positive-direction periodic sub-timescale.

Example 3.3. Consider $\mathbb{T}=\{-4 k, 4 k+3: k \in \mathbb{N}\}$. Note that $\mathbb{T}_{1}=\{-4 k: k \in \mathbb{N}\}$ and $\mathbb{T}_{2}=\{4 k+3: k \in \mathbb{N}\}$ are oriented-direction periodic time scales, i.e., $\mathbb{T}_{1}$ is a negative-direction periodic time scale and $\mathbb{T}_{2}$ is a positivedirection periodic time scale. Hence, $\mathbb{T}$ is a changing-periodic time scale.

Theorem 3.1. If $\mathbb{T}$ is an infinite time scale and the graininess function $\mu: \mathbb{T} \rightarrow \mathbb{R}^{+}$is bounded, then $\mathbb{T}$ is $a$ changing-periodic time scale.

Proof. Without loss of generality, we assume that $\sup \mathbb{T}=+\infty$ and $\inf \mathbb{T}=-\infty$. From Remark 2.1, $\mathbb{T}$ is an oriented-direction intersection time scale. For any $p>0, q<0$, we take some $n_{1}, n_{2}$ such that $p\left\langle n_{1} \bar{\mu}, q\right\rangle$ $-n_{2} \bar{\mu}$, where $\bar{\mu}=\sup _{t \in \mathbb{T}} \mu(t)$ and $\max \left\{n_{1} \bar{\mu}, n_{2} \bar{\mu}\right\} \gg \bar{\mu}+L, L>0$ is the length of a closed finite interval with the largest length in $\mathbb{T}$. We denote the set

$$
\mathbb{I}=\left\{\mathbb{T} \cap \mathbb{T}^{\tau}: \tau \in\left[-n_{2} \bar{\mu}, q\right] \cup\left[p, n_{1} \bar{\mu}\right]\right\} .
$$

Clearly, II forms a semi-ordered set with respect to the inclusion relation and $\mathbb{I}$ is closed. Denote $\mathbb{I}^{\star}$ any subset of $\mathbb{I}$ and is totally ordered. We consider two cases:

Case (1):

$$
\mathbb{I}^{\star}=\left\{\mathbb{T}_{\tau_{n}} \in \mathbb{I}: \mathbb{T}_{\tau_{n}} \supset \mathbb{T}_{\tau_{n+1}}, n \in \mathbb{N}\right\}, \text { where } \mathbb{T}_{\tau_{n}}:=\mathbb{T} \cap \mathbb{T}^{\tau_{n}},
$$

then $\mathbb{T}_{\tau_{1}} \in \mathbb{I}^{\star} \subset \mathbb{I}$ and $\mathbb{T}_{\tau_{1}}$ is an upper bound of $\mathbb{I}^{\star}$ in $\mathbb{I}$.

Case (2):

$$
\mathbb{I}^{\star}=\left\{\mathbb{T}_{\tau_{n}} \in \mathbb{I}: \mathbb{T}_{\tau_{n}} \subset \mathbb{T}_{\tau_{n+1}}, n \in \mathbb{N}\right\},
$$

then $\lim _{n \rightarrow \infty}\left(\mathbb{T} \cap \mathbb{T}^{\tau_{n}}\right)=\bigcup_{n=1}^{\infty}\left(\mathbb{T} \cap \mathbb{T}^{\tau_{n}}\right)=\mathbb{T} \cap \mathbb{T}^{\tau_{\infty}}$ is an upper bound of $\mathbb{I}^{\star}$. Because $\mathbb{I}$ is closed, then $\mathbb{T} \cap \mathbb{T}^{\tau_{\infty}} \in \mathbb{I}$. According to Zorn's lemma, there exists some $\tau_{0} \in\left[-n_{2} \bar{\mu}, q\right] \cup\left[p, n_{1} \bar{\mu}\right]$ such that $\mathbb{T} \cap \mathbb{T}^{\tau_{0}}$ is the maximum element in $\mathbb{I}$. Note that since $\mu$ is bounded, $\mathbb{T} \cap \mathbb{T}^{\tau_{0}} \neq \emptyset$ and $\sup \left(\mathbb{T} \cap \mathbb{T}^{\tau_{0}}\right)=+\infty, \inf \left(\mathbb{T} \cap \mathbb{T}^{\tau_{0}}\right)=-\infty$.

Now we show that $\mathbb{T}$ is a changing-periodic time scales. We divide the proof into three steps, We divide the proof into the following steps:

Step I. We can find a time scale $\mathbb{T}_{0}^{1}$ such that $\mathbb{T}_{0}^{1} \subset \mathbb{T}$ is the largest periodic sub-timescale in $\mathbb{T}$. For this, we make a continuous translation of $\mathbb{T}$ to find a number $\tau_{1}$ such that $\mathbb{T} \cap \mathbb{T}^{\tau_{1}}:=\mathbb{T}_{1}$ is the maximum. Next, consider a translation of $\mathbb{T}_{1}$ again to find a number $\tau_{2}$ such that $\mathbb{T}_{1} \cap \mathbb{T}_{1}^{\tau_{2}}:=\mathbb{T}_{2}$ is the maximum. Continue this process $n$ times to find a number $\tau_{n}$ such that $\mathbb{T}_{n-1} \cap \mathbb{T}_{n-1}^{\tau_{n}}:=\mathbb{T}_{n}$ is the maximum. This process leads to a decreasing sequence of timescale sets:

$$
\mathbb{T} \supset \mathbb{T}_{1} \supset \mathbb{T}_{2} \supset \ldots \supset \mathbb{T}_{n} \supset \ldots
$$

Hence, it follows that $\lim _{n \rightarrow \infty} \mathbb{T}_{n}=\mathbb{T}_{0}^{1}$. This shows that there exists some $\tau_{0}^{1}$ such that $\left(\mathbb{T}_{0}^{1}\right)^{\tau_{0}^{1}} \subseteq \mathbb{T}_{0}^{1}$ through the translation of this number and $\left(\mathbb{T}_{0}^{1}\right)^{\tau_{0}^{1}}$ is maximum (in fact, if $\left(\mathbb{T}_{0}^{1}\right)^{\tau_{0}^{1}} \subseteq \mathbb{T}_{0}^{1}$, then there exists $\mathbb{T}_{0}^{2}$ such that $\mathbb{T}_{0}^{2}=\left(\mathbb{T}_{0}^{1}\right)^{\tau_{0}^{1}} \cap \mathbb{T}_{0}^{1} \subset \mathbb{T}_{0}^{1}$, this is a contradiction since $\left.\lim _{n \rightarrow \infty} \mathbb{T}_{n}=\mathbb{T}_{0}^{1}\right)$, obviously, this also implies that $\mathbb{T}_{0}^{1}$ is not a finite union of the closed intervals.

Now we claim that $\mathbb{T}_{0}^{1} \neq \emptyset$. In fact, if $\mathbb{T}_{0}^{1}=\emptyset$, then there exists some sufficiently large $n_{0}$ such that $\mathbb{T}_{n_{0}}=\emptyset$, i.e., there exists some sub-timescale $\mathbb{T}_{n_{0}-1} \subset \mathbb{T}$ such that $\mathbb{T}_{n_{0}-1}$ has no intersection with itself through the translation of the number $\tau_{n_{0}}$ and $\mathbb{T}_{n_{0}-1} \cap \mathbb{T}_{n_{0}-1}^{\tau_{n_{0}}}$ is the maximum element in

$$
\mathbb{I}_{n_{0}}=\left\{\mathbb{T}_{n_{0}-1} \cap \mathbb{T}_{n_{0}-1}^{\tau}: \forall \tau \neq 0\right\}=\emptyset,
$$

which means that $\mathbb{T}_{n_{0}-1}$ is a single point set, but this is a contradiction since $\sup \mathbb{T}_{n_{0}-1}=+\infty$, inf $\mathbb{T}_{n_{0}-1}=-\infty$. Therefore, $\mathbb{T}_{0}^{1}$ is a $\tau_{0}^{1}$-periodic sub-timescale whose translation direction is determined by the sign of the number $\tau_{0}^{1}$. 
Step II. For the time scale $\mathbb{T}_{1}^{*}:=\overline{\mathbb{T} \backslash \mathbb{T}_{0}^{1}}$, where $\bar{A}$ denotes the closure of the set $A$, by replacing $\mathbb{T}$ with $\mathbb{T}_{1}^{*}$, and repeating the Step I, we can obtain the periodic sub-timescale $\mathbb{T}_{0}^{2}$. For the time scale $\mathbb{T}_{2}^{\star}:=\overline{\mathbb{T} \backslash\left(\mathbb{T}_{0}^{1} \cup \mathbb{T}_{0}^{2}\right)}$, by replacing $\mathbb{T}$ with $\mathbb{T}_{2}^{*}$, and repeating the Step I, we can obtain the periodic sub-timescale $\mathbb{T}_{0}^{3}$. Similarly, we can obtain $\mathbb{T}_{0}^{4}, \ldots, \mathbb{T}_{0}^{n} \ldots$ Obviously, the timescale sequence $\left\{\mathbb{T}_{0}^{i}\right\}_{i \in \mathbb{Z}^{+}}$is well connected and $\left(\mathbb{T}_{0}^{i} \cap \mathbb{T}_{0}^{j}\right) \backslash\left\{t_{i j 0}^{k}\right\}=\emptyset$ for $i \neq j$, where $\left\{t_{i j 0}^{k}\right\}$ is the connected points set between $\mathbb{T}_{0}^{i}$ and $\mathbb{T}_{0}^{j}$. If for some sufficiently large $n_{0}$, still $\mathbb{T}_{n_{0}}^{\star}=\overline{\mathbb{T} \backslash \bigcup_{i=1}^{n_{0}} \mathbb{T}_{0}^{i}}$ is an infinite time scale, then we repeat the Step I again until the remaining timescale $\mathbb{T} \backslash \bigcup_{i=1}^{\infty} \mathbb{T}_{0}^{i}=\emptyset$, or a finite union of the closed intervals.

Step III. Letting the set $\Pi$ of $\mathbb{T}$ be as

$$
\Pi=\left(\bigcup_{i=1}^{\infty} S_{i}\right) \bigcup R_{0}=\left(\bigcup_{i=1}^{\infty}\left\{n \tau_{0}^{i}, n \in \mathbb{Z}\right\}\right) \bigcup R_{0},
$$

where $R_{0}=\{0\}$ or $\emptyset$, from Steps I and II, it follows that $S_{i} \cap S_{j}=\emptyset$ if $i \neq j$.

From Steps I, II, III, we find $\left(\bigcup_{i=1}^{\infty} \mathbb{T}_{0}^{i}\right) \cup\left(\bigcup_{i=1}^{k}\left[\alpha_{i}, \beta_{i}\right]\right)=\mathbb{T}$, where $k$ is some finite number and $\left[\alpha_{i}, \beta_{i}\right]$ are closed intervals for $i=1,2, \ldots, k$, or $\left(\bigcup_{i=1}^{\infty} \mathbb{T}_{0}^{i}\right)=\mathbb{T}$. Therefore, $\mathbb{T}$ is a changing-periodic time scale. This completes the proof.

Remark 3.2. In $[2,10]$, the authors emphasize that "periodic time scale" implies "oriented-direction periodic time scale", all "periodic time scale" is with "translation direction". The definition we should adopt is Definition 2.3. Note Definition 2.3 in this paper includes Definition 1.1 in [7]. Note Definition 3.7 in [8] is equivalent to Definition 1.1 in [7].

Remark 3.3. For any oriented-direction periodic time scale $\mathbb{T}$, we can add $\{-\infty,+\infty\}$ to the time scale such that inf $\mathbb{T}=-\infty$ and $\sup \mathbb{T}=+\infty$. For example, for the time scale (1.1), we can add $\{-\infty\}$ to the time scale such that inf $\mathbb{T}=-\infty$ if we need, and it is still a positive-direction periodic time scale.

Remark 3.4. From Theorem 3.1, Theorem 2.21 in [2] is true.

Theorem 3.2. Let $\mathbb{T}$ be an arbitrary time scale with $\sup \mathbb{T}=+\infty$, inf $\mathbb{T}=-\infty$. If $\mu: \mathbb{T} \rightarrow \mathbb{R}^{+}$is bounded, then $\mathbb{T}$ contains at least one oriented-direction periodic time scale.

Proof. From Remark 2.1, $\mathbb{T}$ is a bi-direction intersection time scale. For any $p>0, q<0$, we take some $n_{1}, n_{2}$ such that $p<n_{1} \bar{\mu}, q>-n_{2} \bar{\mu}$, where $\bar{\mu}=\sup _{t \in \mathbb{T}} \mu(t)$ and $\max \left\{n_{1} \bar{\mu}, n_{2} \bar{\mu}\right\} \gg \bar{\mu}+L, L>0$ is the length of a closed finite interval with the largest length in $\mathbb{T}$. We denote the set

$$
\mathbb{I}=\left\{\mathbb{T} \cap \mathbb{T}^{\tau}: \tau \in\left[-n_{2} \bar{\mu}, q\right] \cup\left[p, n_{1} \bar{\mu}\right]\right\} .
$$

Clearly, If forms a semi-ordered set with respect to the inclusion relation and $\mathbb{I}$ is closed. Denote $\mathbb{I}^{\star}$ any subset of $\mathbb{I}$ and is totally ordered. We consider two cases:

Case (1):

$$
\mathbb{I}^{\star}=\left\{\mathbb{T}_{\tau_{n}} \in \mathbb{I}: \mathbb{T}_{\tau_{n}} \supset \mathbb{T}_{\tau_{n+1}}, n \in \mathbb{N}\right\}, \text { where } \mathbb{T}_{\tau_{n}}:=\mathbb{T} \cap \mathbb{T}^{\tau_{n}},
$$

then $\mathbb{T}_{\tau_{1}} \in \mathbb{I}^{\star} \subset \mathbb{I}$ and $\mathbb{T}_{\tau_{1}}$ is an upper bound of $\mathbb{I}^{*}$ in $\mathbb{I}$.

Case (2):

$$
\mathbb{I}^{\star}=\left\{\mathbb{T}_{\tau_{n}} \in \mathbb{I}: \mathbb{T}_{\tau_{n}} \subset \mathbb{T}_{\tau_{n+1}}, n \in \mathbb{N}\right\},
$$

then $\lim _{n \rightarrow \infty}\left(\mathbb{T} \cap \mathbb{T}^{\tau_{n}}\right)=\bigcup_{n=1}^{\infty}\left(\mathbb{T} \cap \mathbb{T}^{\tau_{n}}\right)=\mathbb{T} \cap \mathbb{T}^{\tau_{\infty}}$ is an upper bound of $\mathbb{I}^{\star}$. Because $\mathbb{I}$ is closed, then $\mathbb{T} \cap \mathbb{T}^{\tau_{\infty}} \in \mathbb{I}$. According to Zorn's lemma, there exists some $\tau_{0} \in\left[-n_{2} \bar{\mu}, q\right] \cup\left[p, n_{1} \bar{\mu}\right]$ such that $\mathbb{T} \cap \mathbb{T}^{\tau_{0}}$ is the maximum element in $\mathbb{I}$. Note that since $\mu$ is bounded, $\mathbb{T} \cap \mathbb{T}^{\tau_{0}} \neq \emptyset$ and $\sup \left(\mathbb{T} \cap \mathbb{T}^{\tau_{0}}\right)=+\infty$ or $\inf \left(\mathbb{T} \cap \mathbb{T}^{\tau_{0}}\right)=-\infty$.

We can find a time scale $\mathbb{T}_{0}^{1}$ such that $\mathbb{T}_{0}^{1} \subset \mathbb{T}$ is the largest periodic sub-time scale in $\mathbb{T}$. For this, we make a continuous translation of $\mathbb{T}$ to find a number $\tau_{1}$ such that $\mathbb{T} \cap \mathbb{T}^{\tau_{1}}:=\mathbb{T}_{1}$ is the maximum. Next, consider a translation of $\mathbb{T}_{1}$ again to find a number $\tau_{2}$ such that $\mathbb{T}_{1} \cap \mathbb{T}_{1}^{\tau_{2}}:=\mathbb{T}_{2}$ is the maximum. Continue this process 
$n$ times to find a number $\tau_{n}$ such that $\mathbb{T}_{n-1} \cap \mathbb{T}_{n-1}^{\tau_{n}}:=\mathbb{T}_{n}$ is the maximum. This process leads to a decreasing sequence of time scale sets:

$$
\mathbb{T} \supset \mathbb{T}_{1} \supset \mathbb{T}_{2} \supset \ldots \supset \mathbb{T}_{n} \supset \ldots
$$

Hence, it follows that $\lim _{n \rightarrow \infty} \mathbb{T}_{n}=\mathbb{T}_{0}^{1}$. This shows that there exists some $\tau_{0}^{1}$ such that $\left(\mathbb{T}_{0}^{1}\right)^{\tau_{0}^{1}} \subseteq \mathbb{T}_{0}^{1}$ through the translation of this number and $\left(\mathbb{T}_{0}^{1}\right)^{\tau_{0}^{1}}$ is maximum (in fact, if $\left(\mathbb{T}_{0}^{1}\right)^{\tau_{0}^{1}} \subseteq \mathbb{T}_{0}^{1}$, then there exists $\mathbb{T}_{0}^{2}$ such that $\mathbb{T}_{0}^{2}=\left(\mathbb{T}_{0}^{1}\right)^{\tau_{0}^{1}} \cap \mathbb{T}_{0}^{1} \subset \mathbb{T}_{0}^{1}$, this is a contradiction since $\left.\lim _{n \rightarrow \infty} \mathbb{T}_{n}=\mathbb{T}_{0}^{1}\right)$, obviously, this also implies that $\mathbb{T}_{0}^{1}$ is not a finite union of the closed intervals.

Now we claim that $\mathbb{T}_{0}^{1} \neq \emptyset$. In fact, if $\mathbb{T}_{0}^{1}=\emptyset$, then there exists some sufficiently large $n_{0}$ such that $\mathbb{T}_{n_{0}}=\emptyset$, i.e., there exists some sub-timescale $\mathbb{T}_{n_{0}-1} \subset \mathbb{T}$ such that $\mathbb{T}_{n_{0}-1}$ has no intersection with itself through the translation of the number $\tau_{n_{0}}$ and $\mathbb{T}_{n_{0}-1} \cap \mathbb{T}_{n_{0}-1}^{\tau_{n_{0}}}$ is the maximum element in

$$
\mathbb{I}_{n_{0}}=\left\{\mathbb{T}_{n_{0}-1} \cap \mathbb{T}_{n_{0}-1}^{\tau}: \tau \neq 0\right\}=\emptyset,
$$

which means that $\mathbb{T}_{n_{0}-1}$ is a single point set, but this is a contradiction since $\sup \mathbb{T}_{n_{0}-1}=+\infty$, inf $\mathbb{T}_{n_{0}-1}=-\infty$. Therefore, $\mathbb{T}_{0}^{1}$ is a $\tau_{0}^{1}$-periodic sub-timescale whose translation direction is determined by the sign of the number $\tau_{0}^{1}$, that is, $\mathbb{T}_{0}^{1}$ is an invariant under translations unit in $\mathbb{T}$. This completes the proof.

Remark 3.5. From Theorem 2.4 in [10], under the conditions in Theorem 3.2, we obtain that $\mathbb{T}$ contains at least one invariant under translations unit. Note that this invariant under translations unit is with direction (i.e. oriented-periodic time scale).

Example 3.4. Consider the time scale $\mathbb{T}=\{-6 k, 6 k+5: k \in \mathbb{N}\}$. We see that $\mathbb{T}$ contains a positive-direction periodic time scale $\mathbb{T}_{1}=\{6 k+5: k \in \mathbb{N}\}$ and a negative-direction periodic time scale $\mathbb{T}_{2}=\{-6 k: k \in \mathbb{N}\}$, respectively. Note that $\mathbb{T}$ is a changing-periodic time scale.

Next, from Definition 2.3, we can apply our results to dynamic equations and obtain some new results related to changing-periodic time scales under the new concept of periodic time scales (i.e., in the sense of Definition 2.3). In order to make this paper more concise, we will not restate the results from [2] here. One can consult [2] for details.

Consider the following linear dynamic equation on a changing-periodic time scale $\mathbb{T}$ :

$$
x^{\Delta}(t)=A(t) x(t)+f(t)
$$

and its associated homogeneous equation

$$
x^{\Delta}(t)=A(t) x(t),
$$

where $A(t)$ is a local-almost periodic matrix function, and $f(t)$ is a local-almost periodic vector function. Further, we assume that $f(t)$ and $A(t)$ are synchronously local-almost periodic functions.

Definition 3.2. We say the following dynamic equation

$$
x^{\Delta_{\tau_{t}}}(t)=A(t) x(t)+f(t), t \in \mathbb{T}_{\tau_{t}}
$$

and its associated homogeneous equation

$$
x^{\Delta_{\tau_{t}}}(t)=A(t) x(t), t \in \mathbb{T}_{\tau_{t}}
$$

are the local-almost periodic dynamic equations generated by (3.3) and (3.4), respectively, where $\Delta_{\tau_{t}}$ implies the $\Delta$-derivative on the time scale $\mathbb{T}_{\tau_{t}}$. Furthermore, we say all the solutions for (3.5) and (3.6) are the local solutions for (3.3) and (3.4), respectively.

From Theorem 3.5, Corollaries 3.7-3.9, and by considering Definition 2.3, we obtain the following corollaries. 
Corollary 3.1. Let $\mathbb{T}$ be a changing-periodic time scale and $\tau_{t}$ be an index function. If (3.4) admits an exponential dichotomy on the local part $\mathbb{T}_{\tau_{t}}$ with $P_{\tau_{t}}=0$ for all $t \in \mathbb{T}$ and $\mathbb{T}_{\tau_{t}}$ is a positive-direction periodic time scale, then (3.3) has a unique local-almost periodic solution on $\mathbb{T}_{\tau_{t}}$ given by

$$
x(t)=\int_{t}^{+\infty} X(t) X^{-1}\left(\sigma_{\tau_{s}}(s)\right) f(s) \Delta_{\tau_{s}} s .
$$

Remark 3.6. In Corollary 3.1, if (3.4) admits an exponential dichotomy on the local part $\mathbb{T}_{\tau_{t}}$ with $P_{\tau_{t}}=$ I for all $t \in \mathbb{T}$ and $\mathbb{T}_{\tau_{t}}$ is a negative-direction periodic time scale, then (3.3) has a unique local-almost periodic solution on $\mathbb{T}_{\tau_{t}}$ given by

$$
x(t)=\int_{-\infty}^{t} X(t) X^{-1}\left(\sigma_{\tau_{s}}(s)\right) f(s) \Delta_{\tau_{s}} s .
$$

If $\mathbb{T}_{\tau_{t}}$ is a bi-direction periodic time scale, then (3.3) has a unique local-almost periodic solution on $\mathbb{T}_{\tau_{t}}$ of the form (3) in Theorem 3.5 in [2].

Remark 3.7. In fact, one can observe that (3.7) and (3.8) are almost periodic solutions for (3.5), according to Definition 3.2, they are the local-almost periodic solutions for (3.3).

From Definition 2.3, we will give the concept of combinable-almost periodic functions on changing-periodic time scales.

Definition 3.3 ([2]). Let $\mathbb{T}$ be a changing-periodic time scale. If there exists an $\omega_{i_{0}}$-periodic sub-timescale set $\left\{\mathbb{T}_{i_{0}}\right\}_{i_{0} \in I}$ with the same direction such that the periods set $\left\{\omega_{i_{0}}\right\}_{i_{0} \in I}$ have a lowest common multiple $\omega$ and $f$ is almost periodic on $\mathbb{T}_{i_{0}}$ for each $i_{0}$, then $f$ is called a combinable-almost periodic function on $\mathbb{T}$ and $I$ is called $a$ combinable index number set. In fact, $f$ is almost periodic on the $\omega$-periodic sub-timescale $\bigcup_{i_{0} \in I} \mathbb{T}_{i_{0}}$. Further, if $\bigcup_{i_{0} \in I} \mathbb{T}_{i_{0}}=\mathbb{T}$, then $f$ is called the globally combinable-almost periodic function on $\mathbb{T}$.

Remark 3.8. In Definition 3.3, $\left\{\mathbb{T}_{i_{0}}\right\}_{i_{0} \in I}$ with the same direction implies that, $\mathbb{T}_{i_{0}}$ are positive-direction periodic time scales for all $i_{0} \in I$; or $\mathbb{T}_{i_{0}}$ are negative-direction periodic time scales for all $i_{0} \in I$.

Remark 3.9. Denote $I=\left\{\tau_{t}: \mathbb{T}_{\tau_{t}}\right.$ are with the same direction and a common periodicity $\omega$ for all $\left.t \in \mathbb{T}\right\}$ and $\mathbb{T}_{I}:=\bigcup_{\tau_{t} \in I} \mathbb{T}_{\tau_{t}}$. One can easily observe that $\mathbb{T}_{I}$ is an $\omega$ oriented-direction periodic sub-timescale of $\mathbb{T}$ and $\mathbb{T}_{I}$ is dependent on $t$.

Definition 3.4. We say the following dynamic equation

$$
x^{\Delta_{c}}(t)=A(t) x(t)+f(t), t \in \mathbb{T}_{I}
$$

and its associated homogeneous equation

$$
x^{\Delta_{c}}(t)=A(t) x(t), t \in \mathbb{T}_{I}
$$

are the combinable-almost periodic dynamic equations generated by (3.3) and (3.4), respectively, where $\Delta_{c}$ implies the $\Delta$-derivative on the time scale $\mathbb{T}_{I}$. Furthermore, we say all the solutions for (3.9) and (3.10) are the combinable solutions for (3.3) and (3.4), respectively.

Corollary 3.2. Let $\mathbb{T}$ be a changing-periodic time scale and $f$ be a combinable-almost periodic function on $\mathbb{T}$, and I be a combinable index number set. If (3.4) admits an exponential dichotomy on the local part $\mathbb{T}_{\tau_{t}}$ with $P_{\tau_{t}}=0$ for all $t \in \mathbb{T}$ and $\bigcup_{\tau_{t} \in I} \mathbb{T}_{\tau_{t}}$ is a positive-direction periodic time scale, then (3.3) has a unique combinablealmost periodic solution on $\bigcup_{\tau_{t} \in I} \mathbb{T}_{\tau_{t}}$ given by

$$
x(t)=\int_{t}^{+\infty} X(t) X^{-1}\left(\sigma_{c}(s)\right) f(s) \Delta_{c} s .
$$


Remark 3.10. In Corollary 3.2, if $\bigcup_{\tau_{t} \in I} \mathbb{T}_{\tau_{t}}$ is a negative-direction periodic time scale, then (3.3) has a unique combinable-almost periodic solution on $\bigcup_{\tau_{t} \in I} \mathbb{T}_{\tau_{t}}$ given by

$$
x(t)=\int_{-\infty}^{t} X(t) X^{-1}\left(\sigma_{c}(s)\right) f(s) \Delta_{c} s .
$$

If $\bigcup_{\tau_{t} \in I} \mathbb{T}_{\tau_{t}}$ is a bi-direction periodic time scale, then (3.3) has a unique combinable-almost periodic solution on $\cup_{\tau_{t} \in I} \mathbb{T}_{\tau_{t}}$ of the form in Corollary 3.8 in [2].

Remark 3.11. In fact, one can observe that (3.11) and (3.12) are almost periodic solutions for (3.9), according to Definition 3.4, they are the combinable-almost periodic solutions for (3.3).

\section{Conclusion}

In this paper, we introduce the concept of periodic time scales with a translation direction, i.e., Definition 2.3. This paper not only provides some new improvements for periodic time scales but also improves the results for almost periodic time scales. In addition, new results related to changing-periodic time scales are obtained. Also, we make several remarks to clarify our new theory.

\section{Further discussion and future work}

Recently, Wang and Agarwal have introduced a series of new concepts on time scales. It is necessary to attach directions to periodic and almost periodic time scales during their translations so that some new results can be obtained. We introduce Definition 2.3 in this paper and update some important results on time scales. From Definition 2.3, we can divide an arbitrary time scale with bounded graininess function $\mu$ into a countable union of periodic time scales. In our future work, we will study periodicity and almost periodicity for dynamic equations on various types of time scales.

Competing interests: The authors declare that they have no competing interests.

Author's contributions: All authors contributed equally to the manuscript and typed, read and approved the final manuscript.

Acknowledgement: This work is supported by Tian Yuan Fund of NSFC (No. 11526181), Yunnan Province Education Department Scientific Research Fund Project in China (No. 2014Y008), and Yunnan Province Science and Technology Department Applied Basic Research Project in China (No. 2014FB102).

\section{References}

[1] M. Bohner, A. Peterson, Dynamic Equations on Time Scales: An Introduction with Applications, Birkhäuser Boston Inc., Boston, 2001.

[2] C. Wang, R.P. Agarwal, Changing-periodic time scales and decomposition theorems of time scales with applications to functions with local almost periodicity and automorphy, Adv. Differ. Equ., 296 (2015) 1-21.

[3] C. Wang, Almost periodic solutions of impulsive BAM neural networks with variable delays on time scales, Commun. Nonlinear Sci. Numer. Simulat., 19 (2014) 2828-2842.

[4] C. Wang, R.P. Agarwal, A classification of time scales and analysis of the general delays on time scales with applications, Math. Meth. Appl. Sci., 39 (2016) 1568-1590. 
[5] C. Wang, R.P. Agarwal, Uniformly rd-piecewise almost periodic functions with applications to the analysis of impulsive $\Delta$ dynamic system on time scales, Appl. Math. Comput., 259 (2015) 271-292.

[6] C. Wang, R.P. Agarwal, A further study of almost periodic time scales with some notes and applications, Abstr. Appl. Anal., (2014) 1-11 (Article ID 267384).

[7] E.R. Kaufmann, Y.N. Raffoul, Periodic solutions for a neutral nonlinear dynamical equation on a time scale, J. Math. Anal. Appl., 319 (2006) 315-325.

[8] Y. Li, C. Wang, Uniformly almost periodic functions and almost periodic solutions to dynamic equations on time scales, Abstr. Appl. Anal., (2011). 22p (Article ID 341520).

[9] M. Adıvar, A new periodicity concept for time scales, Math. Slovaca, 63 (2013) 817-828.

[10] C. Wang, R.P. Agarwal, Relatively dense sets, corrected uniformly almost periodic functions on time scales, and generalizations, Adv. Differ. Equ., 312 (2015) 1-9.

[11] A. Wilansky, Topics in Functional Analysis, Springer, Lecture Notes in Mathematics, Volume 45 (1967).

[12] Y. Li, B. Li, Almost periodic time scales and almost periodic functions on time scales, J. Appl. Math., (2015) 1-8 (Article ID 730672).

[13] B. Sendov, Hausdorff Approximations, Kluwer Academic Publishers, Netherlands, (1990). 